\title{
Sistemas de detección de somnolencia en conductores: inicio, desarrollo y futuro
}

\section{Driver drowsiness detection systems: Beginning, development and future}

\author{
María Agustina Garcés ${ }^{[1]}$, José De Jesus Salgado ${ }^{[2]}$, Jesus Andres Cruz ${ }^{[3]}$ y William Henry Cañón ${ }^{[4]}$
}

\begin{abstract}
Resumen
Muchas son las causas de accidentes de tránsito e industriales a nivel mundial, algunas de estos suceden por errores humanos y otros por fallas mecánicas. El hombre en su afán de proteger vidas, ha inventado sistemas que minimicen el impacto de estos accidentes, pero más que disminuir el daño ahora se piensa en la prevención de los mismos. Uno de los errores humanos más comunes que terminan en accidentes es cuando el conductor u operario industrial es víctima de fatiga y/o somnolencia. Las investigaciones sobre este tema que comenzaron hace 60 años han dejado a través del tiempo novedosos sistemas que permiten determinar el estado de somnolencia de las personas usando técnicas de visión por computador, y un naciente interés por el análisis de señales cerebrales que determinan de forma aún más precisa las diferentes etapas del sueño. En este trabajo se presentan cada una de las técnicas empleadas hasta el momento para detectar somnolencia y su importancia como sistema activo de prevención de accidentes de tránsito e industriales.
\end{abstract}

Palabras clave: Somnolencia, Visión por computador, señales cerebrales, sistemas activos.

Traffic and industrial accidents are caused by many different factors. Some are due to human error and others, mechanical failure. In an effort to protect lives, many systems have been invented to minimize the impact of such accidents; however, prevention is now understood as more important than minimizing damage after the accident has already taken place. Among the most common human errors that lead to accidents is when a driver or industrial operator is overtired, fatigued or feeling drowsy. Research on this subject began 60 years ago and has developed numerous innovative time systems for detecting states of drowsiness in people using computer vision techniques. There has also been a rising interest in the analysis of brain signals that very precisely determine the different stages of sleep. This paper will review each of the techniques used to detect drowsiness and their importance as active prevention systems for traffic and industrial accidents.

Key words: Drowsiness, Computer Vision, brain signals, active systems.

[1] Doctora en Control Automático. Profesora Universidad Nacional de San Juan. San Juan, Argentina, agarces@ gateme.unsj.edu.ar

[2] Magister en Ingeniería Electrónica y de Computadores. Profesor Universidad Surcolombiana. Neiva, Colombia - IEEE Member, josesalgadop@usco.edu.co

[3] Estudiante Ingeniería Electrónica. Presidente Rama Estudiantil IEEE USCO, Universidad Surcolombiana - IEEE Member, eng.j.c@ieee.org

[4] Estudiante Ingeniería Electrónica, Universidad Surcolombiana - IEEE Member, eng.william.c@ieee.org

Recibido: 30 abril 2015 • Aceptado: 25 mayo 2015 


\section{Introducción}

La accidentalidad vial es un problema que aqueja a la población mundial. Según la Organización Mundial de la Salud (OMS) la morbiaccidentalidad (muertes por accidentes de tránsito) es la novena causa de muerte en el mundo (World Health Organization, 2012). Según estadísticas del Fondo de Prevención Vial (FPV), es 11 veces más probable que un accidente deje consecuencias trágicas en las carreteras que en zonas urbanas (Fondo de Prevención Vial, 2009). De igual manera, la Organización Internacional del Trabajo (OIT) dice que cerca del 10\% de los accidentes industriales se deben a errores humanos causados por somnolencia y fatiga laboral (International Labour Organization, 2013).

Las compañías automovilísticas y agencias que vigilan las carreteras y los derechos y deberes de trabajadores concentran sus esfuerzos en salvar el mayor número de vidas posibles bajando al mínimo el número de accidentes que se puedan presentar. Es por esto que se desarrollan técnicas, controles, reglamentaciones laborales y demás medidas que protejan la vida no solo de quien conduce o trabaja en una determinada labor, si no de los que están en su entorno ya que ellos también están en un gran peligro al momento que se presente una falla (Forsman, y otros, 2013).

Desde los años 1950 se desarrollan técnicas de control para minimizar el impacto de los accidentes o prevenirlos (Shaout, y otros, 2011). Es aquí donde se desarrollan sistemas activos y sistemas pasivos para vigilar al conductor u operario en sus labores. Los sistemas pasivos son aquellos que están presentes pero su efecto solo se ve en el momento en el que ocurre el accidente, minimizando el impacto sobre las personas involucradas en el accidente, este es el caso de los Airback (Bolsas de aire) y cinturones de seguridad (Shaout, y otros, 2011). Los sistemas de seguridad activos son en su mayoría electrónicos o electromecánicos, involucran el estudio del comportamiento del conductor u operario, el vehículo u maquinaria y su modo de operación o comportamiento en la carretera, como es un monitoreo constante y en tiempo real se pueden evitar accidentes, lo que resulta más conveniente que minimizar los daños cuando este ya ocurre (Shaout, y otros, 2011). Entre los sistemas activos más destacados está el sistema de velocidad de crucero y los de monitoreo de estado de somnolencia.

El objeto de estudio de este trabajo es mostrar el sistema activo encargado de monitorear el estado de som- nolencia de conductores, maquinistas y demás operarios de vehículos o maquinaria que requieran un estado de vigilia óptimo para llevar a cabo sus labores sin ningún riesgo. Veremos cómo desde hace 60 años se tenía la percepción de llevar a cabo este monitoreo, pero solo se han logrado desarrollos importantes desde los últimos 20 años, y además enmarcaremos nuestra futura investigación en esta área para así acercarnos más a un sistema más eficiente de detección de estado de somnolencia para conductores (Flores, y otros, 2011) (Hachisuka, 2013) (Jo, y otros, 2014).

\section{Somnolencia: causa y efectos}

La somnolencia está definida como la sensación de pesadez y torpeza de los sentidos motivada por el sueño. Esta sensación está determinada como el estado intermedio entre el estado de vigilia y el sueño profundo (Powell, y otros, 2010) (Garcés, y otros, 2014). El comienzo de esta etapa no es un evento único, sino que se origina por cambios en varias funciones neurológicas, se producen cambios sensoriales, en la memoria, en la conciencia, pérdida del pensamiento lógico, latencia en la respuesta a estímulos y alteraciones en los potenciales cerebrales. (Garce?s Correa, 2011)

Se considera que la somnolencia es un estado intermedio entre la vigilia y el sueño profundo, y por esta razón puede ser interrumpido fácilmente por estímulos visuales, auditivos o sensoriales, con lo que la persona puede retornar a un aparente estado de vigilia por un determinado tiempo que dependen de la fatiga y el estado de salud de la persona (Liu, y otros, 2009). Pero, ¿Por qué hablamos de un retorno a un aparente estado de vigilia? Estudios recientes revelan que muchas personas que mantienen un estado de vigilia forzado pueden entrar en estado de somnolencia y mantenerse allí así su comportamiento demuestre lo contrario (Yang, y otros, 2009). La persona puede seguir haciendo sus labores de manera rutinaria, sin mostrar signos físicos de somnolencia, pero debido a que su capacidad de reacción y neurológica se ve seriamente afectada no reaccionara de manera adecuada ante eventos que se presenten de manera súbita.

Aunque se ha mencionado que ciertos estímulos visuales, auditivos y/o sensoriales pueden aparentemente sacar a una persona de su estado de somnolencia, hay otros que podrían empeorar esta situación y ahondar tanto este estado hasta que finalmente la persona caiga en sueño profundo. Cabe recalcar que estos es- 
tímulos no conllevan una estrecha relación entre la somnolencia y sus efectos, más bien son el disparador de un estado presente pero oculto por la persona debido a su estado de fatiga o de salud (Universidad Nacional de Colombia, 2005).

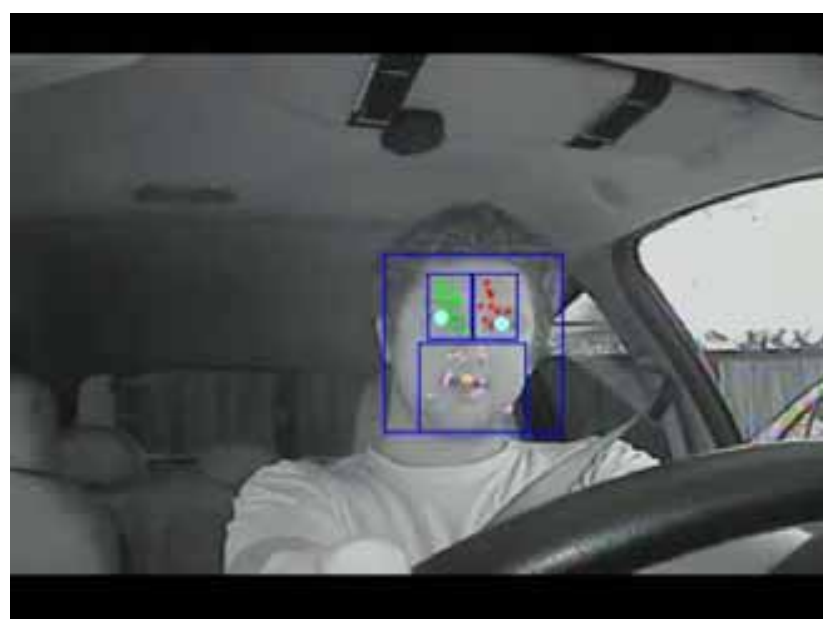

Figura 1. Analisis de visión por computadora del estado de somnolencia (Jimenez-Pinto, y otros, 2009)

Algunas de las características más notorias del estado de somnolencia a nivel fisiológico se pueden ver principalmente en el rostro de las personas, sus ojos, movimientos faciales, de boca y cabeceo. Estos parámetros son claves a la hora de determinar si se está en somnolencia o vigilia. Bostezar repetidamente, dificultad para mantener la mirada en cierto punto, dificultad para mantener la cabeza erguida, el movimiento de las cejas, y dificultad para enfocar son parámetros visibles y contundentes que precisan si una persona está en estado de somnolencia (Hachisuka, 2013). Aunque recientes estudios han demostrado que estos factores no siempre se presentan en trabajadores y conductores que mantienen un estado de vigilia forzado, pues debido a que ellos deben cumplir con determinadas labores y cumplir con una jornada laboral, a veces su estado de somnolencia no puede ser apreciado por métodos de inspección visual (Yang, y otros, 2009).

Otros factores que determinan o dan información clave para saber si se encuentra en un estado de somnolencia son las señales cerebrales, cardiacas y oculografía (Bermúdez, y otros, 2012). A pesar de que estas no se visualizan sin ayuda de instrumentación especial, dan información muy precisa y fiable del estado de somnolencia o vigilia de una persona. Estos datos se pueden obtener por medio de estudios electroencefalográficos que analizan la actividad eléctrica del cerebro, electrocardiográficos que analizan la actividad eléctrica del corazón y electrooculográficos que analizan la actividad eléctrica ocular. >Aquí debemos tener muy en cuenta las fases del sueño NoREM (Non Rapid Eye Moviment - Sin Movimiento Ocular Rápido) (Garce?s Correa, 2011) (Sun, y otros, 2014). El sueño REM (Rapid Eye Moviment - Movimiento Ocular Rápido) no será tenido en cuanta en este estudio, pues su aparición aparte de generar alteraciones irregulares en las señales biológicas que simulan estar en la fase I del sueño NoREM, es un estado de sueño profundo que no es objeto de estudio de esta investigación, pues su presencia es inaceptable de cualquier forma en un operario.

No hay un método o métrica establecida para determinar el estado de somnolencia. Las funciones fisiológicas asociadas con el sueño y sus fases es el único método conocido que da información precisa del estado real de alerta de una persona. Por eso nuestro indicador inicial serán las 4 fases del Sueño NoREM, en cada una de ellas se describe como es la actividad eléctrica cerebral, ocular y arterial y como afecta las funciones corporales. Determinar de forma precisa y eficiente las dos primeras fases del sueño podrá generar las alertas necesarias para evitar muchos accidentes de tránsito e industriales (Alshaqaqi, y otros, 2013).

\section{Sistemas electrónicos de detección de somnolencia}

\subsection{Sistemas de visión por computador}

Actualmente es el método más usado para detectar somnolencia en conductores y operarios de maquinaria pesada, ya tiene aplicaciones comerciales por parte de algunas empresas y es preferido por ser un método no invasivo para el operario y por la sencillez de su implementación. Su desarrollo inicio en la década de 1990, haciéndose más fuerte a mediados y finales, a principios del nuevo milenio, Toyota comenzó a hacer pruebas del sistema en simulaciones cotidianas de conducción para aplicarlo finalmente en 2006 como un sistema embebido en uno de sus modelo Lexus, Mercedes en el 2009 y Ford en el 2010 fueron los siguientes fabricantes en implementar este sistema en algunos de sus modelos (Powell, y otros, 2010) (Shaout, y otros, 2011) (Garce?s Correa, 2011).

Como hemos mencionado en la sección anterior, hay parámetros visibles que permiten determinar si se está pasando de un estado de vigilia a uno de somnolencia, y son estos parámetros los que se digitalizan, pro- 
Tabla 1. Sistemas de detección de somnolencia de visión por computador existentes en el mercado (Shaout, y otros, 2011) (Garcés Correa, 2011).

\begin{tabular}{lll}
\hline Fabricante & Comportamiento a estudiar & Respuesta \\
\hline Ford & Movimiento del vehículo en la carretera & Alertas auditiva y visual \\
\hline Lexus & Ojos del conductor & $\begin{array}{l}\text { NoREM 1: Alertas Visuales y auditivas. NoREM 2. } \\
\text { Frenado del vehículo }\end{array}$ \\
\hline Mercedes & Movimiento del vehículo en la carretera & Alertas auditiva y visual \\
\hline VW & Ojos del conductor & Alertas auditiva y visual \\
\hline Volvo & $\begin{array}{l}\text { Movimiento del vehículo } \\
\text { en la carretera }\end{array}$ & Alertas auditiva y visual \\
\hline
\end{tabular}

cesan y analizan constantemente y en tiempo real con el fin de monitorear al operario. Es por medio de cámaras y sensores en el vehículo que se logran obtener estos datos y generar las alertas necesarias. Las técnicas de visión por computador son el motor que mueve este monitoreo, teniendo una eficiencia de alrededor del $80 \%$, generando alertas necesarias y tempranas para evitar accidentes de tránsito o industriales por un error humano debido a la somnolencia (Forsman, y otros, 2013) (Flores, y otros, 2011) (Lee, y otros, 2011).

Desafortunadamente su implementación no está reglamentada a nivel mundial ni en ningún país como China, India o Estados Unidos que son los principales motores de desarrollo de estas investigaciones. Lo cual permite a trabajadores del sector de la industria como la del transporte de carga, transporte intermunicipal, maquinaria pesada entre otras operar en horarios extendidos lo que conlleva a una inminente fatiga que puede desencadenar en transición de estados de somnolencia, dejando posibles y graves consecuencias para los operarios y los que están en su entorno (Dawson, y otros, 2014) (Jo, y otros, 2014).

Los métodos de visión por computador se centran principalmente en el análisis de las expresiones faciales y movimiento de la cabeza a la hora de conducir, estos métodos toman el rostro y lo modelan en 2D o en 3D, para posteriormente por medio de técnicas matemáticas como regresión lineal o de aprendizaje automático de maquina como las redes neuronales determinar un posible cambio de estado de vigilia a uno de somnolencia.
Hay otros sistemas que adicionalmente analizan el comportamiento del vehículo con respecto a su entorno mientras se está conduciendo (Flores, y otros, 2011) (Alshaqaqi, y otros, 2013). El vehículo cuenta con una cámara en la parte delantera, donde se digitaliza el entorno del vehículo; esto quiere decir la carretera, su dirección y el vehículo por delante de él. Con esto se analiza la reacción del conductor frente a la dirección de su vehículo con respecto a la carretera, velocidad, comportamiento del vehículo frente a baches en la carretera y distancia con respecto al vehículo que esta adelante. Si se detecta un comportamiento anormal en más de uno de estos procesos se procede a generar las alertas necesarias para evitar un accidente de tránsito (García y otros, 2010) (Hachisuka, 2013).

\subsection{Sistemas de análisis de señales bioeléctricas}

Nuestro cuerpo genera diferentes tipos de señales de acuerdo a funciones o estados específicos en el que se encuentre el organismo, estas señales pueden ser debido a la temperatura, presión o cargas bioeléctricas que recorren nuestro organismo. Las señales bioeléctricas se producen por el intercambio de iones entre células a través de su membrana, esto genera una diferencia de potencial medible, que puede ser procesada para brindar información de actividad del sistema nervioso central y el sistema muscular (Alipoor, y otros, 2010) (Alonso, 2011).

Desde los años 1950 la técnica que mide la actividad eléctrica del cerebro (Guzmán, 2005) (Yang, y otros, 2009), es la electroencefalografía (EEG). Esta fue usada para el estudio y la detección de estados de som- 
Tabla 2. Relación de investigaciones sobre la detección de somnolencia analizando señales bioeléctricas

\begin{tabular}{|c|c|c|}
\hline Titulo & Señal analizada & $\mathbf{N}^{0}$. canales y tipo de análisis \\
\hline $\begin{array}{l}\text { EEG assessment of mental fatigue in a driving } \\
\text { simulator (Zhao, y otros, 2012) }\end{array}$ & $\begin{array}{l}\text { Actividad eléctrica del } \\
\text { cerebro y del corazón. }\end{array}$ & $\begin{array}{l}32 \text { canales. } \\
\text { Densidad Espectral de la señal. }\end{array}$ \\
\hline $\begin{array}{l}\text { Evaluation of the workload and drowsiness } \\
\text { during car driving by using high resolution } \\
\text { EEG activity and neurophysiologic indices } \\
\text { (Maglione, y otros, 2014) }\end{array}$ & $\begin{array}{l}\text { Actividad eléctrica cerebral, } \\
\text { cardiaca y ocular. }\end{array}$ & $\begin{array}{l}32 \text { canales. } \\
\text { Densidad Espectral. }\end{array}$ \\
\hline $\begin{array}{l}\text { Combined LVQ Neural Network and } \\
\text { Multivariate Statistical Method Employing } \\
\text { Wavelet Coefficient for EEG Signal } \\
\text { Classification (Kashtiban, y otros, 2011) }\end{array}$ & Actividad eléctrica cerebral. & $\begin{array}{l}32 \text { canales. } \\
\text { Redes Neuronales. }\end{array}$ \\
\hline $\begin{array}{l}\text { Automatic detection of drowsiness in } \\
\text { EEG records based on multimodal analysis } \\
\text { (Garcés, y otros, 2014) }\end{array}$ & Actividad eléctrica cerebral & $\begin{array}{l}1 \text { canal. } \\
\text { Redes Neuronales. }\end{array}$ \\
\hline $\begin{array}{l}\text { Can SVM be used for automatic EEG } \\
\text { detection of drowsiness during car }\end{array}$ & Actividad eléctrica cerebral & $\begin{array}{l}16 \text { canales } \\
\text { Máquinas de soporte vectorial } \\
\text { driving (Yeo, y otros, 2009). }\end{array}$ \\
\hline
\end{tabular}

nolencia en muchas ocasiones. A través del EEG se estudiaron las 5 fases del sueño, 4 NoREM (sin movimiento ocular rápido) y 1 REM (Movimiento ocular rápido) que ayudaron hasta la actualidad a monitorear la actividad cerebral en diferentes actividades cotidianas del hombre como por ejemplo dormir (MIN, 2007).

A finales de la década de 1990 y principios de la década del 2000, diferentes investigadores alrededor del mundo comenzaron a desarrollar técnicas para llevar a cabo un monitoreo EEG a conductores mientras estos realizan sus rutinarias labores, el primero de ellos usaba 32 electrodos y a través de un sistema de Procesamiento Digital de Señales - DSP determinaba si un paciente estaba o no en estado de vigilia, este estudio se llevó a cabo en un laboratorio ambientado con un simulador de conducción, y participaron 30 voluntarios en las pruebas, teniendo una eficiencia del $72 \%$ en la detección temprana de estados de somnolencia (Wilson, y otros, 2000).

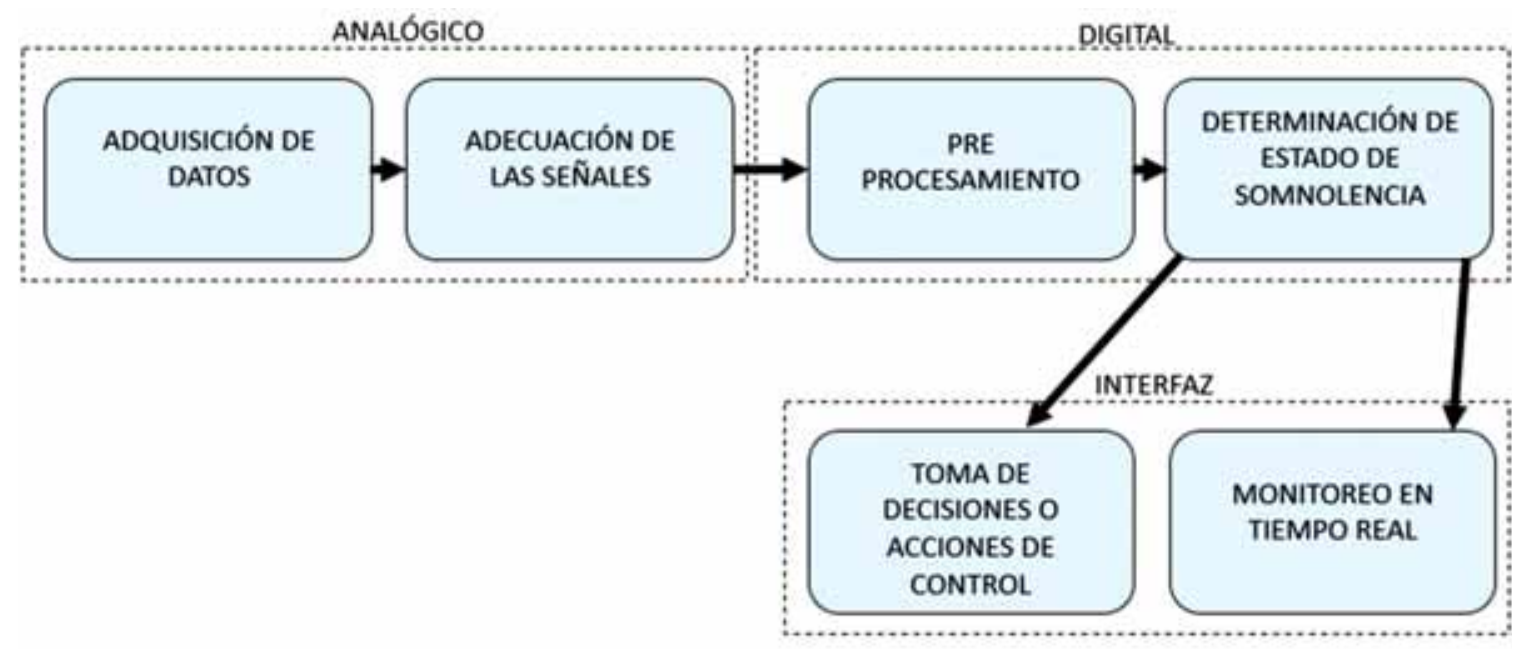

Figura 2. Diagrama de bloques básico de un sistema activo de detección de somnolencia (Yeo, y otros, 2009). 
A medida que los sistemas computaciones se hacían más robustos en el nuevo milenio, el uso de técnicas de aprendizaje automático de maquina en sistemas embebidos se fue popularizando de forma rápida y las investigaciones en el tema fueron tomando cada vez más peso en esta área. El uso de técnicas como las redes neuronales (Kim, y otros, 2012), lógica difusa, redes neurodifusas, aprendizaje profundo y las máquinas de soporte vectorial fueron incursionando en el análisis de señales biomédicas (Yeo, y otros, 2009). El aprendizaje automático de maquina supervisado y no supervisado podía soportarse ahora en microcontroladores, lo que hacía más fácil procesar la señal con equipos portátiles de bajo consumo de potencia (Kim, y otros, 2012).

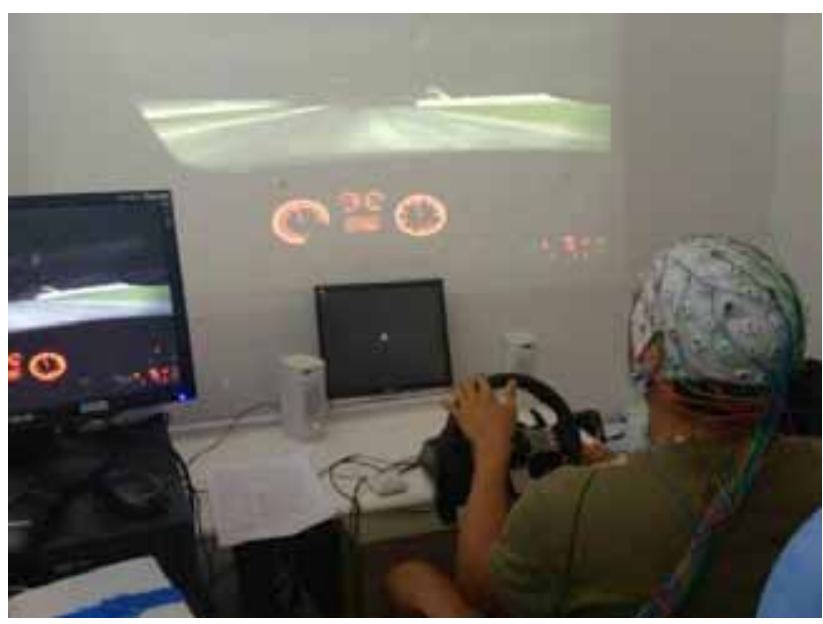

Figura 3. Ambientes simulados para pruebas de sistemas de detección de somnolencia por medio de EEG (Maglione, y otros, 2014)

La primera técnica, y actualmente la más usada para el análisis del estado del sueño fueron las redes neuronales, hay registros de investigaciones desde el año 2000, usando un tratamiento digital de señal por medio de la transformada de Wavelet, y calificándola por medio de una red neuronal cuya salida binaria daba información sobre el estado de somnolencia o vigila de la persona (Wilson, y otros, 2000). Pero fue hasta el 2002 donde se hicieron las primeras clasificaciones utilizando Redes Neuronales para la clasificación de registros de estados del sueño (Yeo, y otros, 2009).

Más recientemente se han realizado investigaciones buscando reducir el número de canales que ofrezcan portabilidad y comodidad a la hora de implementar el monitoreo del sueño por medio de EEG. Recientemente, se ha podido establecer que se pueden adquirir 16 señales diferentes de un solo canal de EEG, entre las cuales se encuentran las señales Alfa, Theta y Delta (Arnin, y otros, 2013) (Garcés, y otros, 2014), las cuales suministran información necesaria sobre los estados del sueño. Por otra parte, la clasificación de las señales EEG ha resultado ser altamente efectiva implementado Maquinas de Soporte Vectorial (Yeo, y otros, 2009), una técnica de aprendizaje automático supervisado que elimina de manera simultánea la clasificación empírica del error y maximiza el margen geométrico en la clasificación, esto quiere decir que se construye un híperplano en donde las muestras son separadas de manera óptima de dos clases con un margen máximo de clasificación, lo que conlleva a minimizar el error de clasificación empírico y aumenta el margen geométrico a la hora de hacer la clasificación.

A la hora de realizar un EEG, no solo se obtiene información o señales del encéfalo, también se obtienen otras señales llamadas artefacto (Akben, y otros, 2010), que no son más que ritmo cardiaco producido por arterias, señales musculares por el movimiento de la cabeza o músculos faciales, y señales eléctricas producidas por oculografía. Estas señales también nos brindan información adicional a la hora de determinar estados de somnolencia, aunque en algunas ocasiones son consideradas ruido y se suprimen en la digitalización y procesamiento de la señal, pero en otras ocasiones estas señales son procesadas para ser analizadas y elevar la eficiencia a la hora de determinar estados de somnolencia (Arnin, y otros, 2013). Aunque realmente analizar estas señales artefacto hasta ahora no incurre en un verdadero aumento de la eficiencia a la hora de llevar a cabo una clasificación de forma confiable, y por el contrario aumenta el uso de recurso computacional y tiempos de ejecución del algoritmo, aun se hacen intentos por dar un uso eficiente a estas señales artefacto. Hasta el momento solo las señales cerebrales Alfa, theta y Delta son suficientes para dar de forma confiable información sobre las fases del sueño (Dias, y otros, 2007).

\section{Conclusiones}

Los sistemas electrónicos embebidos en vehículos son una herramienta esencial para prevenir accidentes de tránsito, pues con ellos se puede llevar a cabo un monitoreo constante y en tiempo real sobre el automotor, el conductor y sus acciones. Más específicamente el sistema de detección de somnolencia adaptado para vehículos constituye una herramienta fundamental para aplicaciones domésticas, de transporte de carga, 
industrial y demás que están sometidos a extensas jornadas de trabajo frente a un volante, generando fatiga, lo que puede llevar a desencadenar diferentes fases de sueño sin control, con consecuencias tan lamentables como la muerte.

Los sistemas de monitoreo del sueño por medio de procesamiento digital de imágenes son una solución primaria y fuerte para poder implementar un sistema que detecte cambios en el estado de vigilia a uno de somnolencia, aunque no es la solución definitiva, pues se ha demostrado que muchas personas pueden estar en alguna de las fases del sueño NoREM y no dar señales aparentes de somnolencia, lo cual reduce significativamente la efectividad de este método para algunas personas que finalmente podrían provocar un accidente de tránsito.

Por otro lado los sistemas que analizan señales bioeléctricas, especialmente las cerebrales, demuestran una mejor confiabilidad a la hora de determinar si una persona está en vigilia o en alguna etapa del sueño NoREM. Esto da una ventaja a este sistema sobre el anterior, sin embargo el uso excesivo de canales de EEG y la incomodidad de usar estos, factores como el sudor, exceso de cabello, grasa capilar y ruido a la hora de procesar la señal es un problema que aún está en etapa de desarrollo. Los sistemas de monitoreo activo que demuestran más eficacia son aquellos que monitorean directamente el comportamiento del organismo del individuo, y por eso es importante que las investigaciones futuras apunten a dar solución a los problemas actuales y presentes en este campo.

\section{Referencias bibliográficas}

1. Akben S.B., SubaşA. y Kıymık M.K. Comparison of artificial neural network and support vector machine classification methods in diagnosis of migraine by using EEG [Conferencia] // Signal Processing and Communications Applications Conference (SIU), 2010 IEEE 18th. 2010, pp. 637-640. DOI: 10.1109/ SIU.2010.5651470.

2. Alipoor M., Pooyan M. y Suratgar Amir Abolfazl Classification of EEG signals in four groups, including healthy subjects with open/closed eyes and epilepsy subjects with/without seizure by PSD estimate (using the multitaper method) and ANN [Conferencia] // Health Informatics and Bioinformatics (HIBIT), 2010 5th International Symposium on. 201, pp. 98-103. DOI: 10.1109/HIBIT.2010. 5478900.
3. Alonso Luis Fernando Nicolás Clasificación de características de electroencefalogramas en sistemas Brain Computer Interface basados en ritmos sensoriomotores [Informe] : Master's thesis / UNIVERSIDAD DE VALLADOLID. 2011.

4. Alshaqaqi B. [y otros] Driver drowsiness detection system [Conferencia] // Systems, Signal Processing and their Applications (WoSSPA), 2013 8th International Workshop on. 2013, pp. 151-155. DOI: 10.1109/WoSSPA.2013.6602353.

5. Arnin J. [y otros] Wireless-based portable EEG-EOG monitoring for real time drowsiness detection [Conferencia] // Engineering in Medicine and Biology Society (EMBC), 2013 35th Annual International Conference of the IEEE. 2013, pp. 4977-4980. ISSN: 1557-170X DOI: 10.1109/EMBC.2013. 6610665.

6. Bermúdez Germán Rodríguez [y otros] Técnicas de reconocimiento de patrones para la clasificación de señales EEG en sistemas BCI [Conferencia] // V Jornadas de Introducción a la investigación en la UPCT 2012/ ed. de Cartagena Universidad Politécnica. 2012, 5(3).

7. Borghini Gianluca [y otros] Measuring neurophysiological signals in aircraft pilots and car drivers for the assessment of mental workload, fatigue and drowsiness [Publicación periódica] // Neuroscience I \& Biobehavioral Reviews. 2014, 44, pp. 58-75. Applied Neuroscience: Models, methods, theories, reviews. A Society of Applied Neuroscience (SAN) special issue. ISSN: 0149-7634 DOI: http://dx.doi. org/10.1016/ j.neubiorev.2012.10.003.

8. Cyganek Bogus $\AA$,aw y Gruszczy $\AA$, ski SÅ, awomir Hybrid computer vision system for drivers' eye recognition and fatigue monitoring [Publicación periódica] // Neurocomputing. 2014, 126, pp. 78-94. Recent trends in Intelligent Data Analysis Selected papers of the The 6th International Conference on Hybrid Artificial Intelligence Systems (HAIS 2011) Online Data Processing Including a selection of papers from the International Conference on Adaptive and Intelligent Systems 2011 (ICAIS 2011). ISSN: 0925-2312 DOI: http://dx.doi.org/10.1016/j.neucom. 2013.01.048.

9. Danisman T. [y otros] Drowsy driver detection system using eye blink patterns [Conferencia] // Machine and Web Intelligence (ICMWI), 2010 International Conference on. 2010, pp. 230-233. DOI: 10.1109/ICMWI.2010.5648121. 
10. Daphne R. Reena y Raj A. Albert ADrowsiness Detection Architecture using Feature Extraction Methodology [Publicación periódica] // Procedia Engineering. 2012, 38, pp. 959-963. $\backslash\{$ INTERNATIONAL $\} \backslash\{$ CONFERENCE $\}$ $\backslash\{\mathrm{ON} \backslash\} \backslash\{$ MODELLING $\} \backslash\{$ OPTIMIZATION $\} \backslash\{$ AND $\backslash\}$ I\{COMPUTING \\}. ISSN: 1877-7058 DOI: http:// dx.doi.org/10.1016/j.proeng.2012. 06.121.

11.Dawson Drew, Searle Amelia K. y Paterson Jessica L. Look before you (s)leep: Evaluating the use of fatigue detection technologies within a fatigue risk management system for the road transport industry [Publicación periódica] // Sleep Medicine Reviews. 2014, 2, 18, pp. 141-152. ISSN: 1087-0792 DOI: http://dx.doi.org/10.1016/j.smrv.2013. 03.003.

12. Delgado Saa J.F. y Cetin M. Discriminative Methods for Classification of Asynchronous Imaginary Motor Tasks From EEG Data [Publicación periódica] / / Neural Systems and Rehabilitation Engineering, IEEE Transactions on. Sept de 2013, 5, 21, pp. 716-724. ISSN: 1534-4320 DOI: 10.1109/TNSRE.2013. 2268194.

13.Dias N.S. [y otros] Comparison of EEG Pattern Classification Methods for Brain-Computer Interfaces [Conferencia] // Engineering in Medicine and Biology Society, 2007. EMBS 2007. 29th Annual International Conference of the IEEE. 2007, pp. 2540-2543. ISSN: 1557-170X DOI: 10.1109/IEMBS. 2007.4352846.

14.Flores M.J., Armingol J.M. y de la Escalera A. Driver drowsiness detection system under infrared illumination for an intelligent vehicle [Publicación periódica] // Intelligent Transport Systems, IET. December de 2011, 4, 5, pp. 241-251. ISSN: 1751956X DOI: 10.1049/iet-its.2009.0090.

15.Fondo de Prevención Vial VII Encuentro Nacional de Secretarios y Autoridades de Transito // VII Encuentro Nacional de Secretarios y Autoridades de Transito. 2009.

16. Forsman Pia M. [y otros] Efficient driver drowsiness detection at moderate levels of drowsiness [Publicación periódica] // Accident Analysis $1 \&$ Prevention. 2013, 0, 50, pp. 341-350. ISSN: 0001-4575 DOI: http://dx.doi.org/10.1016/j.aap.2012.05.005.

17.Friedrichs F. y Yang Bin Camera-based drowsiness reference for driver state classification under real driving conditions [Conferencia] // Intelligent Vehicles
Symposium (IV), 2010 IEEE. 2010, pp. 101-106. ISSN: 1931-0587 DOI: 10.1109/IVS.2010.5548039.

18. Garcés Agustina, Orosco Lorena y Laciar Eric Automatic detection of drowsiness in $\backslash\{E E G \backslash\}$ records based on multimodal analysis [Publicación periódica] // Medical Engineering \\& Physics . 2014, 2, 36, pp. 244-249. ISSN: 1350-4533 DOI: http:// dx.doi.org/10.1016/j.medengphy.2013.07.011.

19. Garce?s Correa A. Procesamiento de Señales cerebrales para la Detección de Somnolencia en Conductores. [Informe] : Ph.D. dissertation / Universidad Nacional de San Juan, 2011.

20.Garcia I. [y otros] Vision-based drowsiness detector for a realistic driving simulator [Conferencia] // Intelligent Transportation Systems (ITSC), 2010 13th International IEEE Conference on. 2010, pp. 887-894. ISSN: 2153-0009 DOI: 10.1109/ITSC. 2010.5625097.

21. Garrett D. [y otros] Comparison of linear, nonlinear, and feature selection methods for EEG signal classification [Publicación periódica] // Neural Systems and Rehabilitation Engineering, IEEE Transactions on. June de 2003, 2, 11, pp. 141-144. ISSN: 1534-4320 DOI: 10.1109/TNSRE.2003. 814441.

22. Guzmán J. J. La Actividad Cerebral // La Actividad Cerebral. 2005.

23. Hachisuka S. Human and Vehicle-Driver Drowsiness Detection by Facial Expression [Conferencia] // Biometrics and Kansei Engineering (ICBAKE), 2013 International Conference on. 2013, pp. 320-326. DOI: 10.1109/ICBAKE.2013.89.

24.Hong Tianyi y Qin Huabiao Drivers drowsiness detection in embedded system [Conferencia] // Vehicular Electronics and Safety, 2007. ICVES. IEEE International Conference on. 2007, pp. 1-5. DOI: 10.1109/ICVES.2007.4456381.

25.Hosseini S.A. y Khalilzadeh M.A. Emotional Stress Recognition System Using EEG and Psychophysiological Signals: Using New Labelling Process of EEG Signals in Emotional Stress State [Conferencia] // Biomedical Engineering and Computer Science (ICBECS), 2010 International Conference on. 2010, pp. 1-6. DOI: 10.1109/ICBECS.2010. 5462520. 
26. International Labour Organization Causas de accidentes laborales: Las alteraciones del sueño en los accidentes // Causas de accidentes laborales: Las alteraciones del sueño en los accidentes. 2013.

27. Jimenez-Pinto J. y Torres-Torriti M. Driver alert state and fatigue detection by salient points analysis [Conferencia] // Systems, Man and Cybernetics, 2009. SMC 2009. IEEE International Conference on. 2009, pp. 455-461. ISSN: 1062-922X DOI: 10.1109/ ICSMC.2009.5346760.

28.Jo Jaeik [y otros] Detecting driver drowsiness using feature-level fusion and user-specific classification [Publicación periódica] // Expert Systems with Applications. 2014, 4, Part 1: Vol. 41, pp. 1139-1152. ISSN: 0957-4174 DOI: http://dx.doi.org/10.1016/ j.eswa.2013.07.108.

29.Kashtiban A.M., Razmi H. y Kozehkonan M.K. Combined LVQ neural network and multivariate statistical method employing wavelet coefficient for EEG signal classification [Conferencia] // Mechatronics (ICM), 2011 IEEE International Conference on. 2011, pp. 809-814. DOI: 10.1109/ICMECH.2011. 5971225.

30. Kim Dajeong [y otros] Detection of drowsiness with eyes open using EEG-based power spectrum analysis [Conferencia] // Strategic Technology (IFOST), 2012 7th International Forum on. 2012, pp. 1-4. DOI: 10.1109/IFOST.2012.6357815.

31.Lee B.-G., Jung S.-J. y Chung W.-Y. Real-time physiological and vision monitoring of vehicle driver for non-intrusive drowsiness detection [Publicación periódica] // Communications, IET. - November de 2011, 17, 5, pp. 2461-2469. ISSN: 1751-8628 DOI: 10.1049/iet-com.2010.0925.

32.Li Xin, Cui Wei y Li Changwu Research on classification method of wavelet entropy and Fuzzy Neural Networks for motor imagery EEG [Conferencia] // Modelling, Identification Control (ICMIC), 2012 Proceedings of International Conference on. 2012, pp. 478-482.

33.Lin Chin-Teng [y otros] A Real-Time Wireless Brain Computer Interface System for Drowsiness Detection [Publicación periódica] // Biomedical Circuits and Systems, IEEE Transactions on. - Aug de 2010. 4, 4, pp. 214-222. ISSN: 1932-4545 DOI: 10.1109/TBCAS.2010.2046415.
34.Liu Charles C., Hosking Simon G. y LennÃ $@$ Michael G. Predicting driver drowsiness using vehicle measures: Recent insights and future challenges [Publicación periódica] // Journal of Safety Research . 2009, 4, 40, pp. 239-245. ISSN: 0022-4375 DOI: http://dx.doi.org/10.1016/j.jsr.2009.04.005.

35. Maglione A. [y otros] Evaluation of the workload and drowsiness during car driving by using high resolution EEG activity and neurophysiologic indices [Conferencia] // Engineering in Medicine and Biology Society (EMBC), 2014 36th Annual International Conference of the IEEE. 2014, pp. 6238-6241. ISSN: 1557-170X DOI: 10.1109/EMBC.2014. 6945054.

36. Masala G.L. y Grosso E. Real time detection of driver attention: Emerging solutions based on robust iconic classifiers and dictionary of poses [Publicación periódica] // Transportation Research Part C: Emerging Technologies. 2014, 0, 49, pp. 32-42. ISSN: 0968090X DOI: http://dx.doi.org/10.1016/j.trc.2014.10.005.

37. MCKEOWN MARTIN J. [y otros] A new method for detecting state changes in the EEG: exploratory application to sleep data [Publicación periódica] // Journal of Sleep Research. 1998, 7, pp. 48-56.

38. MIN MERVYN YEO VEE AN EEG BASED STUDY OF UNINTENTIONAL SLEEP ONSET [Informe] : Ph.D. dissertation / NATIONAL UNIVERSITY OF SINGAPORE. 2007.

39.Phothisonothai M. y Nakagawa Masahiro EEG signal classification method based on fractal features and neural network [Conferencia] // Engineering in Medicine and Biology Society, 2008. EMBS 2008. 30th Annual International Conference of the IEEE. 2008, pp. 3880-3883. ISSN: 1557-170X DOI: 10.1109/ IEMBS.2008.4650057.

40.Picot A., Charbonnier Sylvie y Caplier A. On-Line Detection of Drowsiness Using Brain and Visual Information [Publicación periódica] // Systems, Man and Cybernetics, Part A: Systems and Humans, IEEE Transactions on. - May de 2012, 3, 42, pp. 764775. ISSN: 1083-4427 DOI: 10.1109/TSMCA.2011. 2164242.

41.Powell Nelson B. y Chau Jason K.M. Sleepy Driving [Publicación periódica] // Medical Clinics of North America. 2010, 3, 94, pp. 531-540. Sleep Medicine. ISSN: 0025-7125 DOI: http://dx.doi.org/10.1016/ j.mcna.2010.02.002. 
42.Principe J.C., Gala S.K. y Chang T.G. Sleep staging automaton based on the theory of evidence [Publicación periódica] // Biomedical Engineering, IEEE Transactions on. May de 1989, 5, 36, pp. 503-509. ISSN: 0018-9294 DOI: 10.1109/10.24251.

43. Sabet M. [y otros] A new system for driver drowsiness and distraction detection [Conferencia] // Electrical Engineering (ICEE), 2012 20th Iranian Conference on. 2012, pp. 1247-1251. DOI: 10.1109/ IranianCEE.2012.6292547.

44. Shaout A., Colella D. y Awad S. Advanced Driver Assistance Systems - Past, present and future [Conferencia] // Computer Engineering Conference (ICENCO), 2011 Seventh International. 2011, pp. 72-82. DOI: 10.1109/ICENCO.2011.6153935.

45. Sun Shiliang y Zhou Jin A review of adaptive feature extraction and classification methods for EEG-based brain-computer interfaces [Conferencia] // Neural Networks (IJCNN), 2014 International Joint Conference on. 2014, pp. 1746-1753. DOI: 10.1109/ IJCNN.2014.6889525.

46. Universidad Nacional de Colombia Trastornos del Sueño // Trastornos del Sueño. Bogotá: Lecciones Medicina, 2005. Vol. Capítulo 5.

47. Villar Shirley Cordova, Oviedo Willian A. Perez y Gonzalez Avid Román Implementation of EEG signal processing methods for communication and control application [Publicación periódica] // Revista ECIPerú. October de 2013, 1, 10, pp. 24-33.

48. Wang Boyu [y otros] Comparison of different classification methods for EEG-based brain computer interfaces: A case study [Conferencia] //
Information and Automation, 2009. ICIA'09. International Conference on. 2009, pp. 1416-1421. DOI: 10.1109/ICINFA.2009.5205138.

49. Wang Zhihua [y otros] Study of signal processing system for Electroencephalogram based on TMS320LF2407 [Conferencia] // Automation Congress, 2008. WAC 2008. World. 2008, pp. 1-4.

50.Wilson B.J. y Bracewell T.D. Alertness monitor using neural networks for EEG analysis [Conferencia] // Neural Networks for Signal Processing X, 2000. Proceedings of the 2000 IEEE Signal Processing Society Workshop. 2000, 2, pp. 814-820. ISSN: 1089-3555 DOI: 10.1109/NNSP.2000.890161.

51. World Health Organization Top 10 de las causas de muerte // Top 10 de las causas de muerte. 2012.

52. Yang Ji Hyun [y otros] Detection of Driver Fatigue Caused by Sleep Deprivation [Publicación periódica] // Systems, Man and Cybernetics, Part A: Systems and Humans, IEEE Transactions on. - July de 2009, 4, 39, pp. 694-705. ISSN: 1083-4427 DOI: 10.1109/TSMCA.2009.2018634.

53. Yeo Mervyn V.M. [y otros] Can SVM be used for automatic EEG detection of drowsiness during car driving? [Publicación periódica] // Safety Science. 2009, 1, 47, pp. 115-124. ISSN: 0925-7535 DOI: http://dx.doi.org/10.1016/j.ssci.2008.01.007.

54.Zhao Chunlin [y otros] Electroencephalogram and electrocardiograph assessment of mental fatigue in a driving simulator [Publicación periódica] // Accident Analysis \\& Prevention. 2012, 0, 45, pp. 83-90. ISSN: 0001-4575 DOI: http://dx.doi.org/10.1016/ j.aap.2011.11.019. 